\title{
Heterotrophic potential estimates: an inherent paradox in assuming Michaelis-Menten kinetics
}

\author{
D. F. Smith ${ }^{1}$, W. J. Wiebe ${ }^{2}$ and H. W. Higgins ${ }^{3}$ \\ ${ }^{1}$ Division of Fisheries Research, CSIRO, P.O. Box 20, Marmion, W. A. 6060, Australia \\ ${ }^{2}$ Department of Microbiology, University of Georgia, Athens, Georgia 30602, USA \\ ${ }^{3}$ Division of Oceanography, CSIRO, P. O. Box 21, Cronulla, N. S. W. 2230, Australia
}

\begin{abstract}
Phytoplankton uptake of radioisotope at high substrate concentrations, although frequently invoked to explain the experimental results obtained in heterotrophic potential estimates, can be shown to arise from 2 common mistakes in experimental technique. Experimental results which purportedly support the view of phytoplankton uptake of substrate are invariably obtained if one employs very high specific activity ${ }^{3} \mathrm{H}$-labelled compounds without purification immediately before use. Identical results ensue if the net $\mathrm{cpm}$ of the samples are obtained by subtracting background cpm or the cpm of either 'zero-time' or 'killed' controls, if these have not been counted to the same number of gross counts as the samples themselves.
\end{abstract}

\section{INTRODUCTION}

Interpretation of the data arising from heterotrophic potential experiments employing radioisotopically labelled organic compounds is made particularly difficult by an unfortunate combination of several factors. First, there are some subtle problems associated with the analysis of data transformed to give a straight line. Historically, investigators who originally employed Michaelis-Menten kinetics assumed that both large and small velocities would be measured with the same precision. In heterotrophic potential estimates, however, small velocities are typically associated with samples having low radioactivity (cpm), while large velocities originate from samples having many more cpm. If samples are counted for the same length of time, the total number of counts accumulated will be less for samples of low velocity than for samples of high velocity. Since the precision of the cpm estimates varies by the square root of the total number of counts accumulated, a geometrically varying precision becomes associated with the velocity estimates over the range of low to high velocities. This change in precision has serious consequences if, as is frequently the case, the data are transformed to obtain a straight line graph. In place of a single straight line, which would be the best fit if the data points had equal precision, the graph contains 2 contiguous straight line segments, the one of lesser slope always being seen at the higher substrate concentrations. This artifactual region of lower slope would be indistinguishable from those reported in the literature and ascribed to phytoplankton uptake (e.g. Wright and Hobbie, 1965).

This same artifact also arises if velocities are estimated from sample net cpm and these are obtained by subtracting background $\mathrm{cpm}$ or the radioactivities of zero-time or killed-cell controls if these have been counted to a smaller number of counts than the samples (Smith and Horner, 1981).

Finally, an independent source of an artifactual region of lesser slope in plots of transformed data arises when high specific activity ${ }^{3} \mathrm{H}$-labelled substrates are employed which have not been re-purified just before use. Such preparations are notorious for forming chemicals which are degradation products of the parent compound, particularly if the radiochemical has been stored in the frozen state (for review, see Bayly and Evans, 1968).

The second major obstacle to the unequivocal interpretation of data arising from the experiments is a consequence of assuming that the Michaelis-Menten equation is the appropriate description of radioisotope incorporation by the samples. The incorporation of radioisotope by samples is precisely the same whether the substrate uptake is described by Michaelis-Menten kinetics or follows zero-order kinetics with regard to 
the substrate (Berman and Schoenfeld, 1956). Thus, it is not possible to determine if one has obtained a measure of the ambient substrate concentration $\left(\mathrm{S}_{\mathrm{N}}\right)$ or that value plus a 'transport constant' $\left(\mathrm{K}_{\mathrm{T}}\right)$ from currently available radioisotope incorporation data.

With few exceptions, heterotrophic potential estimates are obtained with techniques that originated with Parsons and Strickland (1962). The theoretical basis of the original technique was generalized to permit independent addition of labelled or unlabelled substrate (Wright and Hobbie, 1965); the technique was subsequently refined to account for losses of label through respiration (Hobbie and Crawford, 1969). Later modifications of the technique employed high specific activity ${ }^{3} \mathrm{H}$-labelled compounds (Azam and Holm-Hansen, 1973; Dietz et al., 1977) to introduce radioisotope to the sample without appreciably elevating the ambient substrate concentration. Inherent in each of the modified techniques is an assumption given in the original proposal of Parsons and Strickland (1962), that the algebraic form of the MichaelisMenten equation is, due to the mechanism of uptake, required to describe radioisotope incorporation by microheterotrophs. It is implicit in the assumption that microheterotrophs are substrate limited in oceanic environments, and that increasing the quantity of labelled substrate administered during an incubation will lead to an increasing rate of radioisotope incorporation at substrate levels below saturation.

In this study we will show that if one makes the opposite assumption, i.e. that the marine microheterotrophs are always substrate saturated and Michaelis-Menten kinetics are never applicable, the expression describing radioisotope incorporation will be indistinguishable from that assuming MichaelisMenten kinetics. We will also show that unless special precautions are taken, application of any of the classical linear transforms to radioisotope incorporation data will produce a graph of two straight line segments, the first, associated with lower substrate concentrations, having a greater slope than the second. This phenomenon will occur if (1) the radiochemical employed contains a degradation product not incorporated by the microheterotrophs, (2) net radioisotope incorporation is obtained by substracting a single blank value from a set of sample values, or (3) net radioactivities are obtained by subtracting a background which has been obtained by accumulating less total counts than the samples.

\section{Basic equations}

The expression describing radioisotope incorporation proposed by Parsons and Strickland (1962) was modified by Wright and Hobbie (1965) to explicitly include a $\mathrm{V}_{\text {Max }}$ term. After simplifying, by excluding the constants required to convert $\mathrm{cpm}$ to $\mathrm{dpm}$ and accounting for isotope effects, the equation is

$$
\frac{\mathrm{Q}_{\mathrm{o}} \mathrm{t}}{\mathrm{Q}_{\mathrm{POC}}}=\frac{\left(\mathrm{K}+\mathrm{S}_{\mathrm{N}}+\mathrm{A}\right)}{\mathrm{V}_{\mathrm{MAX}}}
$$

where $Q_{0}=$ quantity of radioisotope added to the sample $(\mathrm{dpm}) ; Q_{\mathrm{poc}}=$ quantity of radioisotope incorporated into heterotrophs (dpm); $t=$ incubation period (h); $\mathrm{K}=\mathrm{a}$ constant, characteristic of the system and having dimensions of concentration $\left(\mathrm{mg} \mathrm{l}^{-1}\right) ; \mathrm{S}_{\mathrm{N}}=$ ambient concentration of substrate in the water sample $\left(\mathrm{mg} \mathrm{^{-1 }}\right) ; \mathrm{A}=$ sum of the concentrations of added labelled and unlabelled substrate $\left(\mathrm{mg} \mathrm{l}^{-1}\right) ; \mathrm{V}_{\text {MAX }}=$ uptake velocity at substrate saturation $\left(\mathrm{mg} \mathrm{l}^{-1} \mathrm{~h}^{-1}\right)$.

This equation incorporates the assumption of Michaelis-Menten kinetics and has been tranformed for ease of graphical analysis.

If Eq. 1 is expressed in terms of the original experimental variables actually observed and manipulated, it is expressed as

$$
Q_{\mathrm{POC}}=\frac{\mathrm{V}_{\mathrm{MAX}} \mathrm{Q}_{\mathrm{o}} \mathrm{t}}{\left(\mathrm{K}+\mathrm{S}_{\mathrm{N}}+\mathrm{A}\right)}
$$

However, an alternative expression for $Q_{\mathrm{POC}}(\mathrm{t})$ can be derived where we assume only the classic tracer kinetic postulates of Berman and Schoenfeld (1956), i.e. a steady-state system wherein the quantities of label in each compartment are the only variables. In this case the rate of incorporation of label is proportional only to the specific activity of the label in the medium, and the defining equation is

$$
\frac{\mathrm{d}\left(\mathrm{Q}_{\mathrm{POC}}\right)}{\mathrm{dt}}=\frac{\mathrm{HQ}_{\mathrm{o}}}{\mathrm{S}_{\mathrm{T}}}
$$

where $\mathrm{d}\left(\mathrm{Q}_{\mathrm{poc}}\right) / \mathrm{dt}=$ rate of incorporation of radioisotope into heterotrophs $\left(\mathrm{dpm} \mathrm{h}^{-1}\right) ; \mathrm{H}=$ a rate constant characteristic of the system $\left(\mathrm{mg} \mathrm{l}^{-1} \mathrm{~h}^{-1}\right) ; \mathrm{S}_{\Upsilon}=$ total substrate concentration ( $\mathrm{mg} \mathrm{^{-1 }}$ ).

Letting $S_{R}=\left(Q_{0} /\right.$ specific activity $)$, concentration of radioactive compound added to a sample $\left(\mathrm{mg} \mathrm{l}^{-1}\right), \mathrm{S}_{\mathrm{X}}=$ the concentration of unlabelled compound added to a sample $\left(\mathrm{mg} \mathrm{l}^{-1}\right) ; \mathrm{S}_{\mathrm{N}}=$ as for Equation $\left(\mathrm{mg} \mathrm{l}^{-1}\right)$; then

$$
\mathrm{S}_{\mathrm{T}}=\mathrm{S}_{\mathrm{X}}+\mathrm{S}_{\mathrm{N}}+\mathrm{S}_{\mathrm{R}}
$$

The differential equation (Eq. 3) has been solved using the Laplace transform " as it provides insight into the proper choice of a control for such experiments. Solution by the Laplace transform introduces a term $Q_{p o c}(+0)$ which is the radioactivity incorporated into heterotrophs after an incubation period which is arbi-

\footnotetext{
- Laplacian transform of $Q_{\text {POC }}(t)$ is defined as $Q_{\text {POC }}(\omega)$, where $Q_{\mathrm{POC}}(\omega)=\int_{0}^{\infty} Q_{\mathrm{POC}}(\mathrm{t}) \mathrm{e}^{-\omega t} \mathrm{dt}$
} 
trarily short relative to that of the experimental samples. This equation, then is

$$
\omega Q_{\mathrm{POC}}(\omega)-\mathrm{Q}_{\mathrm{POC}}(+0)=\frac{\mathrm{H} Q_{0}}{\mathrm{~S}_{\Upsilon} \omega}
$$

solving for $Q_{P O C}(\omega)$ and applying the inverse operation to obtain $Q_{\text {POC }}$ (t) gives,

$$
Q_{\text {POC }}(t)-Q_{\text {POC }}(+0)=\frac{H Q_{\mathrm{o}} t}{S_{T}}
$$

Expanding the Expression for, $\mathrm{S}_{\mathrm{T}}$, and writing $\mathrm{Q}_{\mathrm{pOC}}(\mathrm{t})$ - $Q_{\text {POC }}(+0)$ as, $Q_{\text {POC }}$ Eq. 6 becomes

$$
Q_{P O C}=\frac{H Q_{o} t}{S_{X}+S_{N}+S_{R}}
$$

Equations (2) and (7) contain an inherent constant of proportionality, i.e. $\mathrm{V}_{\mathrm{MAx}}$ and $\mathrm{H}$ respectively, but Eq. 7 assumes no special significance of $\mathrm{V}_{\text {MAX }}$ as Eq. 2 does. Both derivations contain terms to account for dilution of the added radioisotope by the unlabelled substrate contained in the sample, i.e. in Eq. 1, $\left(S_{N}+K\right)$ as opposed to $\mathrm{S}_{\mathrm{N}}$ in Eq. 7 .

Both derivations contain terms to account for the specific activity of the administered radioisotope, $\mathrm{A}$ in Eq. 2 and $S_{X}+S_{R}$ in Eq. 7. Data arising from a heterotrophic potential experiment will have the same form irrespective of whether the system is described by Eq. 2 or Eq. 7. The interpretations of these data, however, will vary, i.e. $V_{\text {MAx }}$ and $\left(K+S_{N}\right)$ of Eq. 2 will be interpreted as $\mathrm{H}$ and $\mathrm{S}_{\mathrm{N}}$, respectively, if Eq. 7 is invoked. It might seem that the 2 models could be readily distinguished by knowing $S_{X}$ and $S_{R}$ and by chemical measurement of $S_{N}$ then, by using a set of replicate subsamples, measurement of the $\left(\mathrm{Q}_{0} \mathrm{t} / \mathrm{Q}_{\mathrm{poC}}\right)$ intercept value of Eq. 1, in an experiment where A has been made the independent variable. However, this approach contains a potentially serious error. The linear transform of non-linear data demands that the weights given to the linearized data points also be transformed prior to any regression.

The employment of the Michaelis-Menten equation in enzyme kinetics requires the measurement of a number of reaction velocities (v), equivalent to the $\mathrm{Q}_{\mathrm{POC}} / \mathrm{t}$ values of heterotrophic potential estimates. In this situation all the estimates of $v$ could be reasonably assumed to have equivalent standard deviations.

In the case of $Q_{\text {Poc }} / t$ values, however, the standard deviations are frequently a function of the value of $Q_{\text {poc }}$. This occurs if radioactivities are obtained by counting all samples for the same period of time or if the sample net cpm has been obtained by subtracting the radioactivity of a background or control with less total counts accumulated than were accumulated for the samples. Unless the precision of the $\mathrm{Q}_{\mathrm{poc}} / \mathrm{t}$ estimates are equivalent, a bias is introduced to the data, which when transformed, appears as a region of differing slope at higher values of $A$ or $S_{X}$.

To illustrate the consequences of transforming the data and to describe the type of experiments we have been conducting, Eq. 7 was employed to generate the simulated, error-free data set shown in Table 1. The simulated sample radioactivities would be obtained by incubating a set of replicate samples for the same period and with the same quantity of radioisotope. The independent variable is $S_{X}$, the concentrations of unlabelled substrate added to the samples. The results which are obtained are solely a consequence of applying a linear transformation. That is, either Eq. 2 or 7 could have been employed, tracer could have been

Table 1. Effect of background estimates on heterotrophic potential data transformed into a Lineweaver-Burke plot. Theoretical data points were calculated as dpm from the equation $Q_{p o c}\left(S_{X}\right)=H Q_{0} t /\left(S_{R}+S_{N}+S_{X}\right)$ with $100 \%$ counting efficiency, $Q_{0}=6 \times$

\begin{tabular}{|c|c|c|c|c|}
\hline \multirow{3}{*}{$\mathrm{S}_{\mathrm{X}}\left(\mathrm{mg} \mathrm{l^{-1 }}\right)$} & \multirow{3}{*}{ Gross Qpoc (cpm) } & \multicolumn{3}{|c|}{$\left(Q_{0} t /\right.$ Net $\left.Q_{\text {poC }}\right)$ as a function of background substracted } \\
\hline & & \multicolumn{3}{|c|}{ Background (cpm) } \\
\hline & & 25 & 50 & 75 \\
\hline 0.00 & 60050. & 10 . & 10. & 10. \\
\hline 0.01 & 30050. & 20. & 20. & 20. \\
\hline 0.02 & 20050. & 30. & 30. & 30. \\
\hline 0.05 & 10050 & 60. & 60. & 60. \\
\hline 0.10 & 5505. & 109. & 110. & 110. \\
\hline 0.20 & 2907. & 208. & 210. & 212 . \\
\hline 0.50 & 1226. & 500. & 510. & 521. \\
\hline 1.00 & 644. & 969. & 1010. & 1055. \\
\hline 2.00 & 349. & 1852 & 2007. & 2190 \\
\hline 5.00 & 170 & 4138 & 5000. & 6316. \\
\hline 10.00 & 110. & 7059 & 10000 & 17143 \\
\hline
\end{tabular}
$10^{5}, \mathrm{H}=1 \times 10^{-3}, \mathrm{t}=1, \mathrm{~S}_{\mathrm{R}}=\mathrm{S}_{\mathrm{N}}=5 \times 10^{-3}$. A constant background of $50 \mathrm{cpm}$ was added to each datum point to simulate gross $\mathrm{cpm}$. The consequence of calculating $Q_{0} t /$ Net $Q_{P O C}$ with an imprecise background becomes apparent after $S_{X}>10\left(S_{R}+S_{N}\right)$ 
held constant or increased in precise ratio with $S_{X}$ yet the results would be the same.

At values of $S_{X}$, which are of the same order of magnitude as the value of $S_{R}+S_{N}$, the data are insensitive to back ground estimates (Table 1). At larger values of $S_{X}$, the accuracy of the background estimates becomes increasingly important. Too small or too great a background estimate results in plots which appear to consist of 2 straight line segments of differing slopes (Fig. 1). This anomaly is not seen if the smallest values used to indicate some small value of time after addition of the radioisotope. Each radioactivity measurement obtained in the course of an experiment in which $\mathrm{S}_{\mathrm{X}}$ is varied must arise from counting samples until the same number of counts have been accumulated irrespective of the length of time required. All data points will then have equal precision and can be assigned equal weighting during subsequent curve fitting. It has been our practice to employ Eq. 7 and evaluate $\mathrm{H}$ and $\mathrm{S}_{\mathrm{N}}$, by fitting values of $Q_{P O C} / Q_{0} t$ to the corresponding values

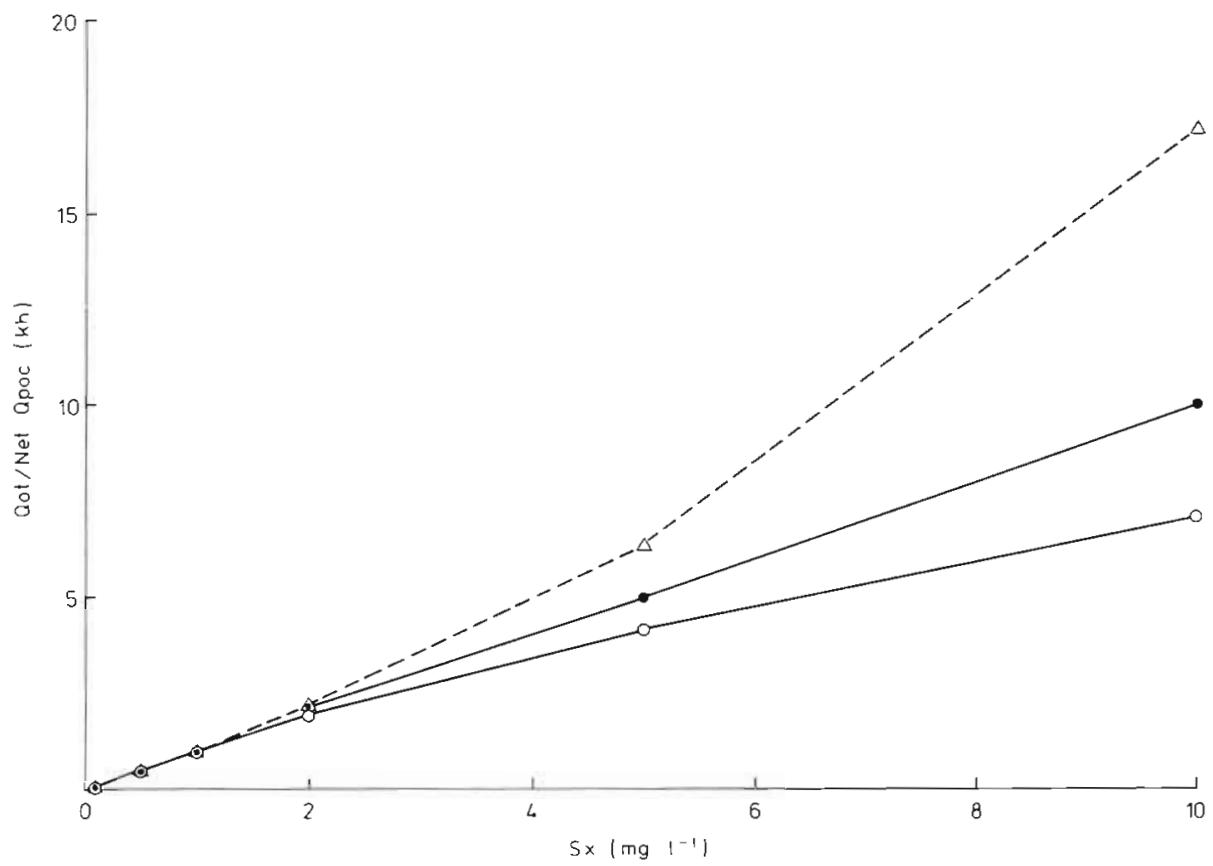

Fig. 1. Artifactual second slope region in Lineweaver-Burke plots of heterotrophic potential caused by a $25 \mathrm{cpm}$ error in background estimates. Shallow curvilinear nature of the deviations are obscured if slight experimental errors are associated with the simulated data points. - background $=50 \mathrm{dpm}$ (no error); 0 - background estimate $=25 \mathrm{cpm}_{i} \Delta$-background estimate $=75 \mathrm{cpm}$

of $\mathrm{S}_{\mathrm{X}}$ employed are large compared to $\mathrm{S}_{\mathrm{R}}+\mathrm{S}_{\mathrm{N}}$, but inaccuracy of background estimates will make evaluation of the intercept value difficult (Fig. 2).

To avoid these difficulties we have developed an experimental technique to obtain precisely the data required by either Eq. 2 or 7, i.e. estimates of the radioactivity on a filter due solely to the incubation of microheterotrophs for a time, $t$, with labelled substrate. A water sample is divided into a set of duplicates and a different quantity of unlabelled substrate, $\mathrm{S}_{\mathrm{X}}$, is added to each pair. The same quantity of labelled substrate, $Q_{0}$, is added to each of the samples and one member of each pair, used as a 'zero-time' control, is filtered immediately, the second is filtered after incubating for some time period, $t$, with the labelled substrate.

The value of $Q_{\text {poC }}$ is obtained by subtracting the radioactivity of the former from the latter member of each pair. The appropriateness of a 'zero-time' control can be rigorously demonstrated from a mathematical view as it arises in consequence of the properties of the Laplacian operator (Aseltine, 1958). The symbol used in Eq. 5, QpoC $(+0)$, is standard mathematical notation of $S_{X}$, using $\operatorname{SAAM}^{*}$, a program permitting multiple non-linear regressions.

The data can be transformed and the $Q_{0} t / Q_{\text {PoC }}$ and $\mathrm{S}_{\mathrm{X}}$ values fitted to the reciprocal of $\mathrm{Eq}_{\mathrm{q}} 7$ if the standard deviation $\sigma_{L i}$ of the data points is transformed (Bevington, 1969) by the relation

$$
\sigma_{\mathrm{Li}}=\sigma_{\mathrm{i}} /\left(Q_{\mathrm{poC}} / \mathrm{Q}_{\mathrm{o}} \mathrm{t}\right)
$$

where, $\sigma_{i}=$ standard deviation of the ith observed value; $\sigma_{\mathrm{Li}}=$ transformed deviation of the ith transformed value.

\section{MATERIAL AND METHODS}

Surface seawater samples were collected in 201 polyethylene buckets and transported to the laboratory within $20 \mathrm{~min}$. Replicate $50 \mathrm{ml}$ portions of sample were dispensed into $50 \mathrm{ml}$ polycarbonate centrifuge tubes

\footnotetext{
- Mathematical Research Branch, National Institute of Arthritis and Metabolic Diseases, National Institutes of Health, Bethesda, Maryland 20014, USA
} 


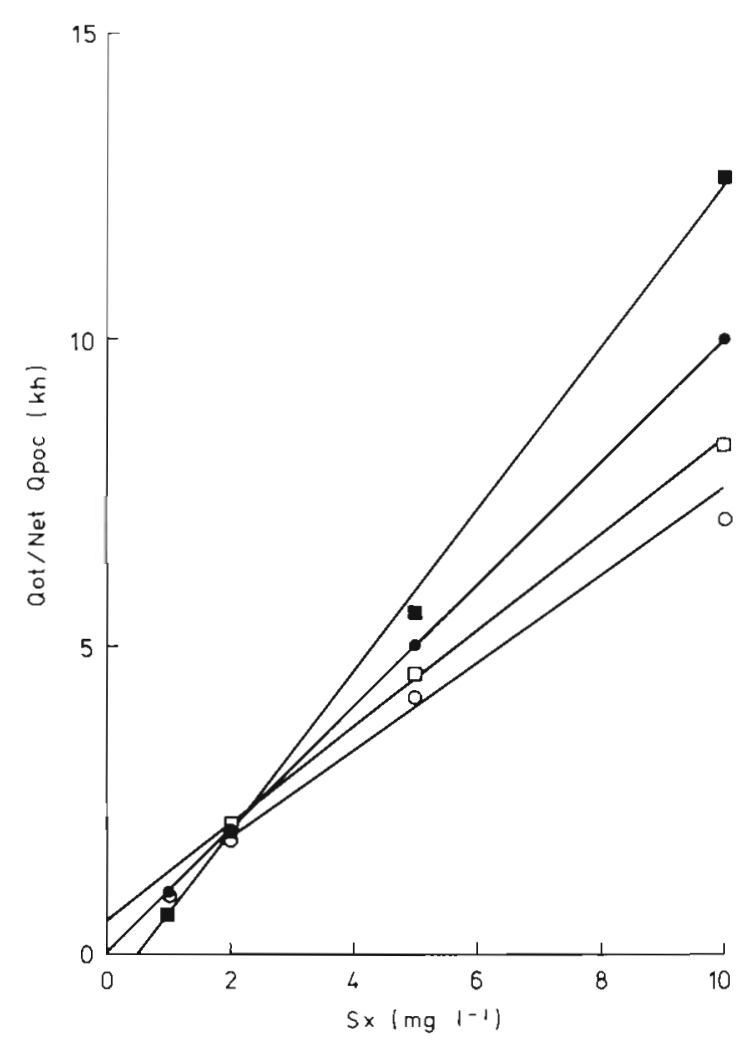

Fig. 2. Sensitivity of $S_{N}$ estimates to precision of background measurements. Extrapolations to obtain $S_{N}$ values after linear transformations of data are notoriously imprecise. Each of the 4 line segments was obtained from the same simulated, errorfree, data set of gross counts by assuming different values of background before obtaining the sample net counts. Backgrounds: $0-25 \mathrm{dpm} ; \square-37.5 \mathrm{dpm}_{\mathrm{i}} \bullet-50 \mathrm{dpm}$ (no error); $\square-$ $62.5 \mathrm{dpm}$

with screw caps or, in some experiments, $10 \mathrm{ml}$ portions were dispensed to counting vials with screw cap lids. A (l-x)ml portion of membrane filtered seawater (MFSW) made $113 \mu \mathrm{M}$ in D-glucose or $10 \mu \mathrm{M}$ in each of the l-amino acids, alanine, arginine, aspartic, glutamic, glycine, isoleucine, leucine, lysine, phenyalanine, proline, serine, threonine, tyrosine, and valine
(A-grade, Calbiochem San Diego, Calif., USA) was added to each sample. By dispensing an additional $\mathrm{x}$ $\mathrm{ml}$ of membrane filtered seawater (MFSW) (pore size $0.2 \mu \mathrm{m}$ ) each sample received a total volume of $1.0 \mathrm{ml}$. Carrier solution (CS) was prepared that contained the same amino acids, or D-glucose, but was $500 \mathrm{mM}$ in concentration. The same quantity of radioisotope was introduced to each tube as D-[ ${ }^{14} \mathrm{C}$ (U)] glucose $(11.6$ $\mathrm{MBq} / \mathrm{m}$ mol, New England Nuclear) or as an equimolar equal specific activity mixture $(370 \mathrm{MBq} / \mathrm{m} \mathrm{mol})$ of labelled amino acids (Radiochemical Centre, Amersham, Sydney, NSW Australia) in quantities ranging from 18.5 to $37-\mathrm{kg} \mathrm{kBq}$, depending on the experiment.

At timed intervals, samples were filtered on membrane filters which had been pooled and kept in a beaker of $\mathrm{CS}\left(\mathrm{T}=0^{\circ} \mathrm{C}\right)$ during the experiment. Prior to filtering each sample, a $1.0 \mathrm{ml}$ portion of $\mathrm{CS}$ was filtered, the vacuum $(<20 \mathrm{kPa})$ released, and a second $1.0 \mathrm{ml}$ portion of CS placed on the filter and the sample added. Filters were dried overnight in vacuo over $\mathrm{KOH}$ then transferred to counting vials containing $15 \mathrm{ml}$ of Permablend ${ }^{\circledR}$ (Packard Instruments, Downers Grove, Ill. USA). Unless otherwise noted, samples were counted in a Packard Model 3360 liquid scintillation counter. All samples were counted until 20,000 counts had been accumulated and sample count rates converted to $\mathrm{dpm}$ by the method of external standardization.

\section{RESULTS}

In one experiment (Table 2) replicate water samples were incubated in the dark at ambient seawater temperature $\left(20^{\circ} \mathrm{C}\right)$. The same quantity of radioisotope $(18.5 \mathrm{kBq})$ but varying amounts of the unlabelled amino acid mixture were added to each sample. For each concentration of unlabelled amino acid mixture employed, the radioactivity in a sample filtered immediately after the addition of tracer, i.e. the 'zero'

Table 2. Radioactivity incorporated by microheterotrophs incubated with labelled amino acid mixture of decreasing specific activity

\begin{tabular}{|c|c|c|c|c|c|}
\hline \multirow{2}{*}{$\begin{array}{l}S_{x} \\
\quad\left(m g ~^{-1}\right)\end{array}$} & \multicolumn{2}{|c|}{ Gross counts in $Q_{\mathrm{POC}}$} & \multirow{2}{*}{$\begin{array}{l}Q_{P O C} \\
(1)-(2) \\
(\mathrm{cpm})\end{array}$} & \multirow{2}{*}{$\begin{array}{c}Q_{P O C} / t \\
\left(\mathrm{cpm} \mathrm{h}^{-1}\right)\end{array}$} & \multirow{2}{*}{$\begin{array}{c}Q_{0} t / Q_{P O C} \\
\left(h^{-1}\right)\end{array}$} \\
\hline & $\begin{array}{l}\text { Experimental (1) } \\
(\mathrm{cpm})\end{array}$ & $\begin{array}{l}\text { Zero time control (2) } \\
(\mathrm{cpm})\end{array}$ & & & \\
\hline 0.00 & 28952. & 1800. & 27152 & 53135. & 21. \\
\hline 0.01 & 15376. & 1329. & 14047 & 27170 & 41. \\
\hline 0.02 & 7264 . & 698. & 6566. & 12627. & 88. \\
\hline 0.05 & 3557. & 518. & 3039 & 5913. & 188. \\
\hline 0.10 & 1915. & 548. & 1367. & 2639. & 421. \\
\hline 0.20 & 1192. & 486. & 706. & 1347. & 824. \\
\hline 0.50 & 666 & 373. & 293. & 555. & 2000 \\
\hline 1.00 & 447. & 297. & 150. & 281. & 3950. \\
\hline
\end{tabular}




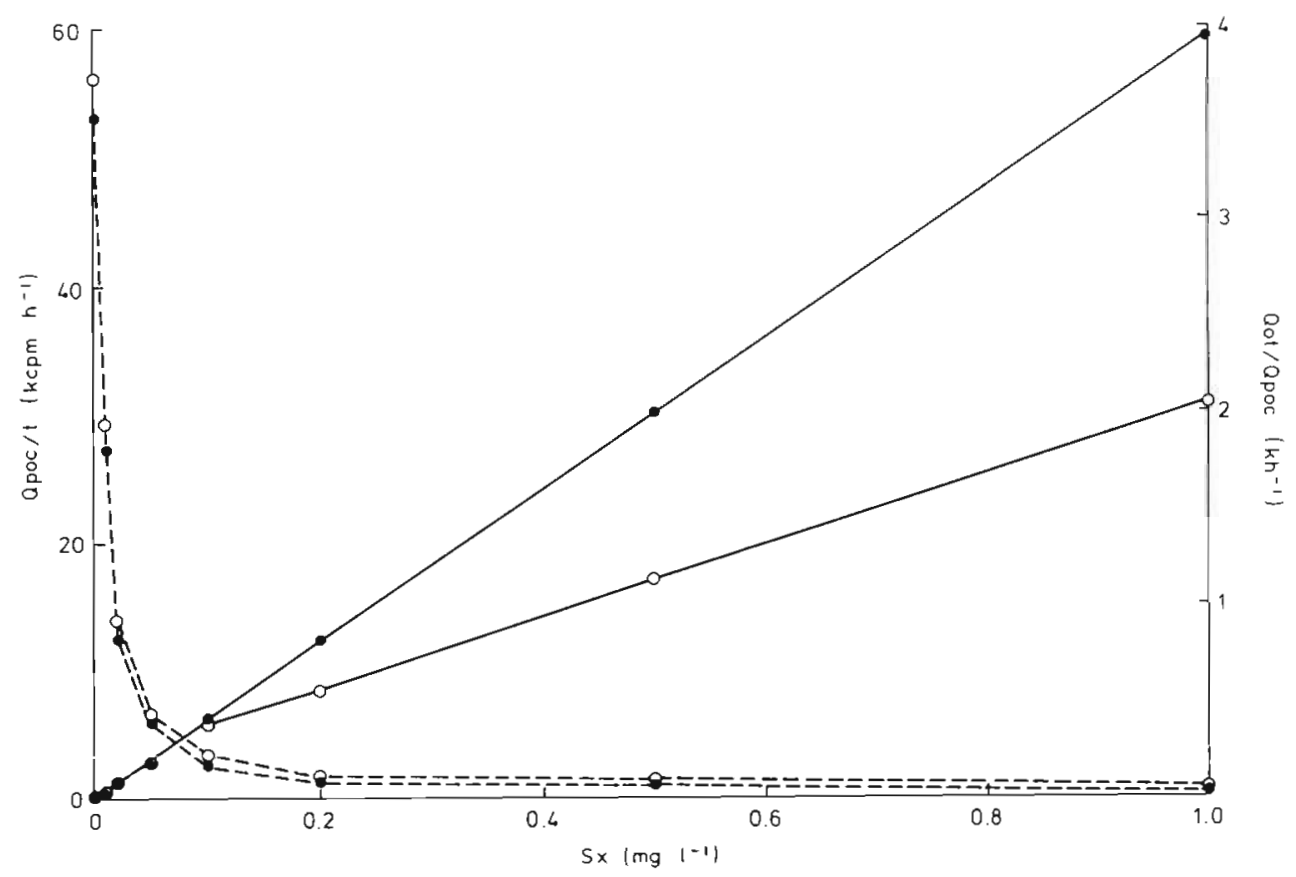

Fig. 3. Effect of background subtraction and the Lineweaver-Burke transformation on radioisotope incorporation data. Radioisotope incorporation rates (Table 2) were obtained by subtracting the radioactivities of zero time controls either from that of the samples ( $\bullet$ or from the net radioactivities of the samples $(O)$. Either approach yields apparently similar results until experimental data plot $(--)$ is transformed into the reciprocal, linear plot $(-)$

time control, was subtracted from the radioactivity of a sample of the corresponding concentration, which had been allowed to incubate for a recorded time (ca. 30 min.). The resultant $Q_{\text {poc }}$ value was divided by the actual incubation period to obtain the rate of uptake Analogous $Q_{\text {poc }}$ values were also obtained by subtracting an average background of $156 \mathrm{cpm}$. The relatively high background was a consequence of employing an ambient temperature liquid scintillation counter (BetaMate, Mark II, Nuclear-Chicago Corp.). Both sets of $Q_{P O C}$ values appeared quite similar (Fig. 3) before taking reciprocal values, i.e. before plotting $Q_{0} t / Q_{P O C}$. The Lineweaver-Burke plot obtained from sample net counts contains an artifact, a lesser slope for values of $S_{x}>0.1$, due to taking the reciprocal of radioactivity estimate made with an imperfect background count. The plot employing $Q_{\mathrm{POC}}$ values obtained by subtracting zero time controls however, shows no change in slope over the entire range of concentrations of $S_{X}\left(0-1.0 \mathrm{mg} \mathrm{l}^{-1}\right)$ employed in the experiment.

The appearance of a region of differing slope in a Lineweaver-Burke plot can occur for reasons other than subtraction of imprecise background estimates. In one experiment employing zero time controls, radioactivity was introduced as $\mathrm{D}-\left[{ }^{14} \mathrm{C}(\mathrm{U})\right]$-Glucose without previous purification (Fig. 4). Instead of decreasing asymptotically to zero, the $Q_{\text {PoC }} / t$ values approached a limit of $8130 \mathrm{cpm} \mathrm{h}^{-1}$, suggesting that the observed radioactivities were the sum of those due to incorporation of D-glucose of decreasing specific activity plus a compound whose specific activity was constant. The effect of the radioactive impurity upon the resultant data is, after transformation to obtain a LineweaverBurke plot, the generation of two straight line segments having different slopes (Fig. 4).

A TLC chromatogram (No. 13255, Eastman Kodak Co., Rochester New York, USA ) of the labelled Dglucose on cellulose showed 2 spots of radioactivity after being developed with n-butanol, EtOH, $\mathrm{H}_{2} \mathrm{O}$ $(50: 30: 20 \mathrm{v} / \mathrm{v})$. The faster moving spot contained $80 \%$ of the applied radioisotope and, after eluting, cochromatographed with D-glucose. In subsequent experiments employing labelled D-glucose with authenticated radiochemical purity, $Q_{0} t / Q_{P O C}$ values decreased asymptotically to zero. In two such experiments (Table 3) 'ambient' D-glucose concentrations were experimentally altered. A single water sample received 3 discrete additions of an unlabelled D-glucose solution; after each addition, a portion was removed and employed as an independent water sample in a subsequent heterotrophic potential measurement. The addition of D-glucose produced no discernible increase in the rate constant, $\mathrm{H}$, although at the highest substrate concentration employed, $6.46 \mathrm{mg} \mathrm{l}^{-1}$, a decrease was apparent.

\section{DISCUSSION AND CONCLUSIONS}

Although the most widely employed method of determining heterotrophic potential estimates invokes the parameters $K_{M}$ and $V_{\text {Max }}$, the applicability of Michaelis-Menten kinetics to natural populations is irrelevant. Whether one assumes uptake velocity of the total substrate to be constant or to increase with sub- 
Fig. 4. Consequence of employing a ${ }^{14} \mathrm{C}$-labelled $\mathrm{D}$ glucose preparation without prior chromatography. Radioactive impurities were not subject to isotope dilution as only increasing amounts of unlabelled Dglucose were added to the replicate samples. The non-zero asymptote, obtained by adding the same quantity of label and varying quantities of carrier to each sample (•) indicates the presence of such an artifact by information not obtained if both radioisotope and carrier are simultaneously varied. The consequence of transforming such data into a Lineweaver-Burke plot (o) is to generate 2 straight line segments of differing slopes

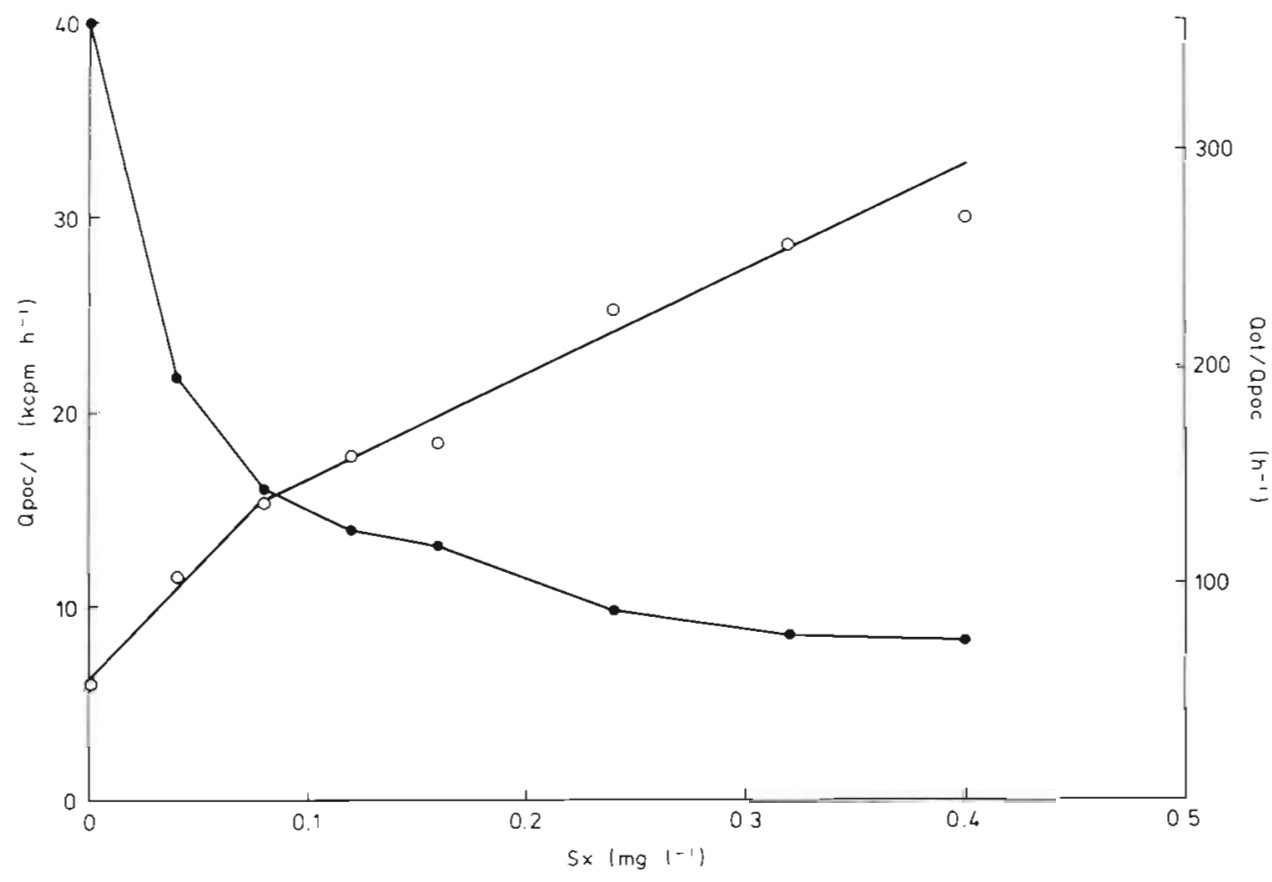

Table 3. Effect of D-glucose addition upon estimating heterotrophic potential. D-glucose was added to a water sample in 3 discrete steps. After each addition a portion of the water sample was removed and employed in an experiment to estimate $\mathrm{S}_{\mathrm{N}}$ and $\mathrm{H}$

\begin{tabular}{|lccc|}
\hline Experiment & $\begin{array}{c}\text { Added Glucose } \\
\left(\mathrm{mg} \mathrm{l}^{-1}\right)\end{array}$ & $\begin{array}{c}\mathrm{S}_{\mathrm{N}} \\
\left(\mathrm{mg} \mathrm{l}^{-1}\right)\end{array}$ & $\begin{array}{c}\mathrm{H} \\
\left(\mathrm{mg} \mathrm{l}^{-1} \mathrm{~h}^{-1}\right)\end{array}$ \\
\hline \multirow{3}{*}{1} & 0.06 & 0.02 & $3.12 \times 10^{-4}$ \\
& 0.12 & 0.12 & $3.41 \times 10^{-4}$ \\
& 0.39 & 0.21 & $3.13 \times 10^{-4}$ \\
2 & 0.13 & 0.14 & $2.02 \times 10^{-4}$ \\
& 1.95 & 2.72 & $1.99 \times 10^{-4}$ \\
& 6.46 & 5.78 & $1.47 \times 10^{-4}$ \\
\hline
\end{tabular}

strate concentration the results obtained with a radioisotopic determination of heterotrophic potential will be the same. Although Eq. 2 and 7 are identical, with $V_{\text {MAX }}$ replaced by $H$, and $A$ by $S_{X}+S_{R}$, the interpretations placed upon the experimental results differ. If $V_{\text {MAX }}$ is known, the value of $\left[Q_{0} t / Q_{\text {Poc }}\right]_{A-o}$ allows one to calculate the quantity $\mathrm{K}_{\mathrm{M}}+\mathrm{S}_{\mathrm{N}}$ from Eq. 2 or, given the analogous experimental quantities, $S_{N}$ from Eq. 7.

Experimental verification of the presence or absence of the term $K_{M}$ is not possible with the techniques commonly employed, not only because of the ambiguity in the assigned value of $\left[\mathrm{Q}_{\mathrm{O}} \mathrm{t} / \mathrm{Q}_{\mathrm{POC}}\right]_{\mathrm{A}-\mathrm{O}}$ arising from statistical data point error, but also because of an approximation made in both Eq. 2 and 7 . In both equations, each value of $Q_{P O C} / t$ is assumed to represent, adequately, the rate of radioisotope uptake at time $t_{i}$ this is valid only if radioisotope uptake rate is

constant. Although the rate of carbon uptake may indeed be constant during an experiment, rate of radioisotope uptake is frequently not. Wiebe and Smith (1977) demonstrated this and showed that confusion between carbon flux and radiocarbon incorporation has resulted in many workers measuring only a pool size divided by an incubation period of arbitrary length although attempting to measure rates of dissolved organic carbon production by phytoplankton. The proper evaluation of $\mathrm{dQ}_{\mathrm{poC}} / \mathrm{dt}$ required, in conjunction with accurate $S_{N}$ determinations, to demonstrate a nonzero value of $K$, is a set of time varying radioisotope samples for each of a set of $\mathrm{S}_{\mathrm{X}}$ values (Berman and Schoenfeld, 1956; Smith, 1974).

Wright and Hobbie (1965) postulated that heterotrophic uptake by phytoplankton could account for the departure of their data from the classical LineweaverBurke plot at total substrate concentrations greater than $0.58 \mathrm{mg} \mathrm{l}^{-1}$. An examination of their $\mathrm{cpm}$ values in this substrate range reveals that the graph in question will return to a normal Lineweaver-Burke plot by subtracting an average of $112 \mathrm{cpm}$ from the count data they employed. The net cpm which they plotted were the differences between incubated samples and a single sample to which radioisotope was added and immediately fixed with Lugol solution. Employing one sample as a blank, i.e. subtracting a constant from each experimental value will, if an error term exists, result in precisely the departure from linearity which they observed.

The obvious criticism that a fixed sample cannot serve as a control for an unfixed sample, can be avoided by employing a zero time control. Our support 
of such controls in these types of experiments arises from the necessity of a control to differ from an experimental sample in only one property. It is interesting that the employment of a zero time control is also explicitly stated in the solution of the Laplacian equation.

Although the frequently employed technique of heterotrophic potential estimation proposed by Parsons and Strickland (1962) assumes that MichaelisMenten kinetics describe the uptake of substrate by marine micro-heterotrophs, the results obtained by employing this technique or its subsequent modifications can afford no proof as to the validity of such an assumption. Our derivation asumes the contrary, i.e. that microheterotroph populations are substrate saturated at ambient concentrations, yet it leads to an expression for radioisotope incorporation that is indistinguishable from that of Parsons and Strickland (1962). Since the experimental results are the same by either approach we are left with the question of what are the metabolic states of marine microheterotrophs.

Employment of the Lineweaver-Burke transformation to obtain the Michaelis-Menten parameters $K_{M}$ and $V_{\text {MAX }}$ has been discouraged for some time even in studies of enzyme kinetics (Lee and Wilson, 1971; Glick, et al. 1979). There are further restrictions upon its employment in heterotrophic potential studies where smaller velocities can be associated with lower precisions of estimates. This association will always occur with samples of varying radioactivities that have been counted for the same period of time or with data which contain a term originating from a blank determined with less precision than the samples. This artifact is sufficient to account for the results interpreted by Wright and Hobbie (1965) as being due to phytoplankton uptake at higher substrate concentrations.

In order to assess properly measurements of heterotrophic potential, which are essentially reports of parameter estimates obtained by fitting data points to an equation, the following information is required:

1) Reports should include the equation invoked to fit the observed data and any transformed equation employed in the curve fitting.

2) The method of assigning relative weights to the data points should be given along with the transform employed for the weights, if the fitting was to a transformed equation.
(3) Criteria used in halting the curve fitting program, minimization of sums of squares, etc., should be stated along with the usual criteria of variance of the parameter estimates. Presentation of these elementary criteria of data analysis are as necessary to the reader in evaluating the results presented as the details of the radioisotope techniques employed.

\section{LITERATURE CITED}

Aseltine, J. A. (1958). Transform method in linear system analysis. McGraw-Hill, New York

Azam, F., Holm-Hansen, O. (1973). Use of tritiated substrates in the study of heterotrophy in seawater. Mar. Biol. 23: 191-196

Bayì, R. J., Evans, E. A. (ed.) (1968). Storage and stability of compounds labelled with radioisotopes. RCC Review 7. The Radiochemical Centre, Amersham, United Kingdom

Berman, M., Schoenfeld, R. (1956). Invariants in experimental data on linear kinetics and the formulation of models. J. appl. Phys. 27: 1361-1370

Bevington, P. R. (1969). Data reduction and error analysis for the physical sciences. McGraw Hill, New York

Dietz, A. S., Albright, L. J., Tuominen, T. (1977). Alternative model and approach for determining micobial heterotrophic activities in aquatic systems. Appl. environ. Microbiol. 33: 817-823

Glick, N., Landman, A. D., Roufogalis, B. D. (1979). Correcting Lineweaver-Burke calculations of $V$ and $K_{M}$. TIBS, Elsevier/North-Holland Biomedical Press, p. 82-83

Hobbie, J. E., Crawford, C. C. (1969) Respiration corrections for bacterial uptake of dissolved organic in natural waters. Limnol. Oceanogr. 14: 528-532

Lee, H.-J., Wilson, I. B. (1971). Enzymic parameters: measurement of $\mathrm{V}$ and $\mathrm{Km}$. Biochim. biophys. Acta 242: 51-522

Parsons, T. R., Strickland, J. D. H. (1962). On the production of particulate organic carbon by heterotrophic processes in seawater. Deep Sea Res. 8: 211-222

Smith, D. F. (1974). Quantitative analysis of the functional relationships existing between ecosystem components. I Analysis of the linear intercomponent mass transfers. Oecologia 16: 97-106

Smith, D. F., Horner, S. M. J. (1981). Tracer kinetic analysis applied to problems in marine biology. In: Platt, T. (ed.) Physiological bases of phytoplankton ecology. Can. Bull. Fish. Aquat. Sci. 210: 113-129

Wiebe, W. J., Smith, D. F. (1977). Direct measurement of dissolved organic carbon release by phytoplankton and incorporation by microheterotrophs. Mar. Biol. 42: $213-223$

Wright, R. T., Hobbie, J. E. (1965) The uptake of organic solutes in lake water. Limnol. Oceanogr. 10: 22-28 